\title{
EL APRENDIZAJE CON EL USO DE LAS NUEVAS TECNOLOGÍAS DE LA INFORMACIÓN Y LAS COMUNICACIONES
}

\author{
RAÚL R. FERNÁNDEZ AEDO* \\ PEDRO MARIO SERVER GARCÍA* \\ ELIANIS CEPERO FADRAGA** \\ Universidad de Ciego de Avila
}

\section{Introducción}

Cuando se habla de nuevas formas de aprendizaje debemos analizar si se trata de cambios e innovaciones en términos de los procesos cognitivos del individuo o de nuevos procedimientos, metodologías y modelos para promover el aprendizaje, aprovechando para ello diversos recursos y estrategias a nuestro alcance, en especial la introducción de las redes que en la educación ha venido a ampliar y acelerar el manejo e intercambio de información y de comunicación y en especial la educación a distancia.

La educación a distancia se ha venido consolidando con el uso de los medios informáticos y de telecomunicaciones como modelos virtuales de educación, que mantienen en común factores como; la no presencia física, el tiempo, el espacio y el modelo educativo.

Un elemento importante es la práctica pedagógica tendiente a generar espacios para producir conocimiento a través de diversos métodos; es decir, es la práctica pedagógica el elemento decisivo para hacer de los nuevos modelos y del uso de las nuevas tecnologías propuestas innovadoras para el aprendizaje, innovaciones educativas además de tecnológicas.

Así, el reto de la educación a distancia o educación virtual, podemos llamarles indistintamente, parece ser la forma de disponer un espacio educativo apoyado en lo tecnológico, para favorecer no la simple reproducción o adquisición del saber sino, por el contrario, las posibilidades de nuevas composiciones y creaciones a partir de las actuales condiciones del saber.

Los entornos de aprendizaje virtuales constituyen una forma totalmente nueva de tecnología educativa y ofrecen una compleja serie de oportunidades y tareas a las instituciones de enseñanza de todo el mundo, el entorno de aprendizaje virtual se define como un programa informático interactivo de carácter pedagógico que posee una capacidad de comunicación integrada.

Los entornos de aprendizaje virtuales son, por tanto, una innovación relativamente reciente y fruto de la convergencia de las tecnologías informáticas y de telecomunicaciones que se ha intensificado durante los últimos diez años.

Los ambientes de aprendizaje son planeados para crear las condiciones pedagógicas y contextuales, donde el conocimiento y sus relaciones con los individuos son el factor principal para formar una "sociedad del conocimiento". Como innovaciones para el aprendizaje en dicha planeación deben atenderse sus componentes: los asesores, tutores o monitores, los estudiantes, los contenidos y su tratamiento o metodología didáctica y los medios tecnológicos.

En la actualidad hay diversas maneras de concebir un ambiente de aprendizaje en la educación formal, que contemplan no solamente los espacios físicos y los medios, sino también los elementos básicos del diseño instruccional. Existen al menos cinco componentes principales que lo 
conforman: el espacio, el estudiante, el asesor, los contenidos educativos y los medios. Por supuesto que no son exclusivos de los ambientes de aprendizaje en modelos no presenciales, cualquier propuesta pedagógica tiene como base estos elementos. Por ello, la planeación de la estrategia didáctica es la que permite una determinada dinámica de relación entre los componentes educativos.

\section{Desarrollo}

Hablar de Nuevas Tecnologías es referirse a la multimedia, la televisión por cable y satélite, al CD-ROM, y a los hipertextos donde su materia prima es la información, se consideran nuevas tecnologías esencialmente las computadoras y los programas informáticos que permiten el acceso a redes, básicamente porque los avances tecnológicos, han dado a la computadora un protagonismo como instrumento pedagógico ya que permite el acceso a grandes cantidades de información.

Son crecientes las investigaciones relacionadas con las redes de comunicación y el correo electrónico. Llama especial atención el énfasis existente en el estudio de la interactividad (particularmente desde entornos educativos), referida a distintos fines, paquetes y formatos de aprendizaje.

En este sentido, una de las clasificaciones más útiles que ha surgido para poder estudiar los medios se refiere a las posibilidades de interacción o "réplica" que presentan. Así, se habla de medios de "una vía" y medios de "dos vías", para diferenciar aquellos que operan bajo un esquema técnico y comunicacional basado en el flujo de información del emisor al receptor, pero no a la inversa, y aquellos que permiten esa reversibilidad.

La interactividad permite el desarrollo de procesos de comunicación e intercambio entre los sujetos rompiendo barreras temporales y espaciales, por tanto, el medio está jugando un papel socializador. Entonces, en los sistemas educativos las computadoras desempeñan principalmente tres funciones: la función tradicional de instrumento para que los alumnos adquieran un nivel mínimo de conocimientos informáticos; la de apoyar y complementar contenidos curriculares; y, la de medio de interacción entre profesores y alumnos, entre los mismos alumnos y entre los propios profesores. La incorporación de medios por consiguiente, obliga a los usuarios a tener una alfabetización tecnológica, lo cual se logra teniendo acceso a lecturas e ideas relacionadas con el uso de la tecnología; adquiriendo un marco de referencia tecnológico amplio que le permita saber por qué está haciendo lo que hace y por qué no hace otras cosas. Es importante que el estudiante y el docente se sientan seguros en su habilidad para apropiarse de la tecnología. Es recomendable que cuando sea posible, reflexionen acerca de su propia experiencia tecnológica, para no caer en la copia de modelos de implementación ajenos.

La alfabetización tecnológica no puede dejar de lado aspectos como el lenguaje, el aprendizaje, el conocimiento y la cultura. En este sentido, ya no será suficiente que los alumnos 
universitarios sepan leer con sentido para interpretar y apropiarse de los conocimientos, tendrán que llegar con habilidades que les permitan otros modos de relacionarse con las nuevas tecnologías, es decir, en sus empatías cognitivas y, expresivas con ellas, y en los nuevos modos de percibir el espacio y el tiempo.

Es cierto que el trabajo en redes genera procesos de interacción y de diálogo entre personas donde la información adquiere nuevos significados mediante el intercambio de mensajes con otros, no debemos olvidar que el medio por sí mismo no hace de los estudiantes mejores aprendices, su incorporación requiere modelos de uso muy claros de manera que permitan la apropiación de los contenidos presentados.

Disponer de equipos y de aplicaciones no es garantía de utilización, ni de que el uso que se haga sea el óptimo, o el más adecuado. Representa para el profesor un trabajo extra en la planificación y gestión de la enseñanza.

Debemos comenzar por entender que la tecnología transforma nuestra relación con el espacio y con el lugar, la tecnología permite volver a localizar el aprendizaje en conexión con el mundo. Esta dispersión de poderes es lo que los expertos señalan como un potencial que brinda esta tecnología al ámbito educativo, ya que los educadores y los estudiantes podrán generar sus propios estilos, modos o maneras de aprender.

El acceso a redes de información en el ámbito escolar es prometedor, sin embargo no debemos dejar de lado la preocupación acerca del tipo de información que circula en ellas al momento de reflexionar sobre las Nuevas Tecnologías.

Con Internet, ha surgido una biblioteca mundial «virtual», interconectada y diseminada geográficamente, cuyos documentos puede consultar cualquier persona que posea una computadora, un mecanismo de comunicación (módem) y un teléfono. Además, distintos usuarios pueden colaborar a distancia en la creación de documentos (para lo que se creó inicialmente la World Wide Web).

No obstante, si no se dispone de instrumentos de navegación eficaces (mapas y brújulas, por así decirlo), los docentes y estudiantes se podrían pasar la vida "navegando" por Internet en busca de esa información.

Si bien es importante saber buscar y localizar bancos de información que enriquezcan y apoyen los procesos de aprendizaje, es necesario replantear las maneras en que los alumnos pueden adquirir conocimientos e informaciones sin perder de vista que en toda situación didáctica el centro deberá ser el estudiante. La función del profesor será la de un facilitador que presta asistencia cuando el estudiante busca conocimientos.

Se trata entonces de nuevos esquemas donde las tecnologías de información y comunicación con apoyo de las telecomunicaciones constituyen un instrumento básico del trabajo intelectual cotidiano. 
La herramienta utilizada es solo un medio para despertar el interés, mantener la motivación y la participación activa en el proceso de enseñanza-aprendizaje.

Es por ello que no debemos ver a la computadora como nuevo objeto mágico que posibilita mejoras importantes en el entorno y las personas. La falta de conexión o coherencia entre los fines y los medios de enseñanza. La interacción cognitiva y emocional del alumnado con los medios se ha venido a denominar la cognición situada.

Es importante dejar en claro que la Internet informa, pero no transforma. El ser humano, es sobre todo búsqueda; espacio de construcción de amplias redes interdisciplinarias, entrelazando fragmentos de un todo, reuniendo lo disperso, elaborando en esa búsqueda su mensaje, original y único, que implica lectura de la realidad, interpretación del mundo y construcción de un sistema de códigos, moldeando con el cerebro, más que con las fibras ópticas el mensaje.

El conjunto de informaciones disponibles en Internet nos desafía a construir una sociedad del conocimiento. Desaparece el don de la verdad, aparece aquel que la consigue, o mejor dicho, aquel que la construye a partir de la información y en su interacción con los demás. Si la búsqueda es un proceso interactivo, rico y dialógico esa búsqueda se transforma en mensaje.

Buscar el mensaje es mucho más que recibirlo. Es construir un sistema de códigos que utilice el lenguaje para expresar la observación de la realidad y compartirla a través de las tecnologías de la comunicación.

Mediante las nuevas tecnologías, y de manera especial con Internet, se tiene acceso a información pero no al conocimiento, para analizar los efectos cognitivos y para promover efectos deseables, debemos considerar además de las potencialidades y limitaciones de cada medio, la propuesta educativa dentro de la cual está inmerso, las actividades de aprendizaje propuestas y los contenidos a abordar.

El aprendizaje ya no es el mismo cuando está soportado con las nuevas tecnologías; el diseño conceptual para introducir estas tecnologías al servicio de la educación es una tarea primordialmente pedagógico-comunicacional.

Ante esto debemos diseñar y evaluar la introducción de nuevas tecnologías no solamente desde su aplicación educativa sino también desde su función comunicativa, debe mirarse el modelo de comunicación que subyace al sistema educativo específico, esto incluye la educación a distancia, la educación para los medios, la educación informal. En todos estos sistemas sucede lo mismo: el aprendizaje se da en la medida en que el individuo se siente involucrado y en este sentido es que el ambiente mediado por tecnologías provoca procesos de aprendizaje, no es la tecnología sino el uso didáctico, combinado con la práctica con/sobre medios.

Los medios son meros vehículos que proporcionan instrucción. Los atributos de un medio son sus capacidades, siempre presentes para ser usadas para influir en el aprendizaje de los estudiantes. 
El debate sobre la influencia de los medios es valioso, porque nos ayuda a clarificar quiénes somos, qué estamos tratando de hacer, qué conocemos y cómo invertir mejor los limitados recursos dedicados a la investigación, parece más productivo considerar la efectividad de métodos que los medios de forma aislada.

La visión de la enseñanza y el aprendizaje que suelen tener en cuenta la mayoría de las personas que producen medios de enseñanza, se sustenta en la idea de que el medio o la planificación de la enseñanza que ellos han desarrollado, si se utiliza de la forma que ellos han pensado, que consideran 'la correcta', logrará que el alumnado adquiera un determinado aprendizaje. En este sentido suelen no tener en cuenta las características intrínsecas del estudiante, su biografía de aprendizaje, las expectativas y capacidades de quien va a interactuar con un medio, produciendo procesos de muy distinto sentido y calidad, en definitiva, el aprendizaje y todo el conglomerado de elementos susceptibles de facilitar o inhibir su proceso de aprendizaje.

La planeación de las propuestas de innovación educativa con apoyo en estas nuevas tecnologías debe considerar, pues, cómo se sitúa el usuario ante la herramienta, qué actividades de aprendizaje realiza, qué valor educativo tienen, qué papel están representando en el proceso de adquisición o elaboración del conocimiento.

El conocimiento supone una relación de acción práctica entre la mente y el mundo.

El aprendizaje supone una iniciación cognitiva simultánea a ciertas actividades de cooperación y práctica múltiple.

El aprendizaje también es el puente entre el conocimiento y la experiencia, ya que cuando la experiencia es comprendida, apropiada, se convierte en una forma especial de conocimiento que genera capacidad para crear información y guiar la experiencia posterior.

Las computadoras, se están convirtiendo en un instrumento que facilita el aprendizaje, en razón de que parece más adaptada a la educación que las tecnologías anteriores, resultando igual o incluso más fácil su empleo, y además posee capacidades de comunicación. El problema o foco de atención son los métodos y enfoques para su mejor aprovechamiento. En general, no se han realizado investigaciones rigurosas que demuestren claramente que los alumnos asimilan un mayor volumen de conocimientos que en los procedimientos pedagógicos habituales, aparte de aprender a utilizar las nuevas tecnologías con distintos objetivos, aunque quizás este último aprendizaje es el que está resultando cada vez más útil en la vida cotidiana fuera de la escuela.

De acuerdo con esta función, los métodos o enfoques pedagógicos preferidos se han definido de formas diversas, como "aprendizaje mediante la experiencia», "aprendizaje mediante la investigación», «aprendizaje mediante el descubrimiento» y «aprendizaje en clase abierta».

Toman mayor relieve conceptos como aprender a aprender, aprender a ser, aprender a hacer y preguntas del tipo: cómo la gente conoce, cómo se percibe a sí misma, cómo usa y comparte 
información, cómo se relaciona con otros y cómo desarrolla sus capacidades para continuar aprendiendo.

El estudio independiente involucra al estudiante en la toma de decisiones sobre el espacio y el tiempo del aprendizaje, la identificación de sus propias necesidades y la auto-instrucción en ambientes en los que no cuenta con la presencia física del profesor.

En modelos no presenciales (a distancia o virtuales), la característica más distintiva en términos del estudiante, es que éste debe tener mayor responsabilidad que en la modalidad de tipo tradicional. El participante debe formarse una idea bien clara de las metas que persigue con el estudio independiente y escoger el programa, los medios, la estrategia de aprendizaje apropiada para lograr estos objetivos propuestos,

Se requiere por parte del estudiante, hoy día, que maneje los nuevos medios que abren otras posibilidades de comunicación como son las computadoras, el uso de Internet como recurso de aprendizaje, el uso de multimedia que integra diferentes lenguajes en un CD-ROM, las teleconferencias. Así mismo y a la par, es necesario que el estudiante despliegue su propia capacidad de generación de comunicaciones multimedia para hacer presentaciones de sus ideas, de su proyecto de investigación, etc.

En los modelos no presenciales con el uso de nuevas tecnologías, la participación en comunidades de aprendizaje es importante porque permite la socialización del conocimiento. En este sentido, el concepto de comunidad dentro de los ambientes virtuales se ha enriquecido, diversificado y ampliado.

Las comunidades pueden surgir, entonces, en función del gusto, de la ocasión o de la ocupación del momento. Las comunidades moldean también a la tecnología acorde con sus necesidades, audiencias e intereses.

Los entornos generados por esas comunidades se caracterizan porque promueven habilidades en individuos y grupos, para acceder, manipular y compartir información que pueda servir para solucionar problemas o crear productos.

Las investigaciones actitudinales suelen estar basadas en la teoría cognitiva que asume como cierto que la motivación influye a la hora de la implicación en una tarea y/o en la calidad del esfuerzo empleado a la hora de aprender. Las actitudes y creencias que tenemos hacia los medios determinan la forma en que interaccionemos con ellos y, en consecuencia, los productos que se obtengan.

Ya habíamos mencionado que una abundancia de información no es necesariamente de gran beneficio por sí misma. De hecho, esta explosión de información ha producido una correspondiente necesidad de procesamiento de información para lograr los resultados deseados.

Estamos hablando entonces de un cambio de paradigma en la educación donde el aprovechamiento pedagógico de las nuevas tecnologías demanda nuevas formas de atención, manejo de nuevos lenguajes, creación de nuevos espacios donde se requiere que el alumno tenga 
autonomía e independencia, para que él pueda administrar su tiempo, diseñar una metodología de estudio.

Un elemento no señalado en párrafos anteriores pero no por ello menos importante es que se tenga una fuerte motivación, el alumno en la modalidad a distancia o virtual, trabaja solo pero no en soledad porque está acompañado permanentemente por el sistema, por los materiales con las recomendaciones para su mejor aprovechamiento, por el apoyo docente, etc.

El alumno necesita tener compromiso con lo que está aprendiendo para llevar adelante las tareas y alcanzar los objetivos propuestos. Así pues, la base del estudio independiente es un sistema motivacional sólido que se adhiera a diversos componentes justificatorios del estudio y el aprendizaje. La motivación se compone de elementos internos y externos que en equilibrio sustentan el estudio. Los elementos externos ayudan a encontrar una dirección adecuada en la actividad concreta, mientras que los elementos internos mantienen el control del estudio.

Por su parte, si el estudiante está inmerso en un programa de educación formal, el sistema le dirá que espera de él, cuál será el rol que él tenga que cumplir, cuáles son las reglas de juego que el programa plantea, qué se espera del alumno en cuanto a estudio independiente y en cuánto al cumplimiento de determinados plazos para abordar un programa o para desarrollar el trabajo, en cuanto a las fechas de evaluación etc.

Lo más importante es que el estudiante sea consciente de su proceso de formación, y en esa medida, que él sepa tomar sus propias decisiones, estamos hablando de un aprendizaje 'autogestivo' donde el estudiante debe conocer sus propios estilos y sus propios modos de aprender, entendiéndolos mejor, podrá potenciarlos. Los usuarios potenciales para esta modalidad son predominantemente adultos, de hecho la modalidad a distancia tiene sus orígenes en la atención de adultos. Los adultos aprenden de manera distinta que los niños y que los jóvenes, en este sentido el adulto es responsable de sus propios aprendizajes, dispone de experiencias que facilitan y enriquecen su aprendizaje. Puede aprender lo que quiera pero necesita más tiempo para hacerlo, busca la aplicabilidad de sus conocimientos. Posee cuatro intereses fundamentales para estudiar: incentivo económico, capacitación profesional, deseo de continuar estudios superiores y socializarse. Es propicio al diálogo en un plano de igualdad con docentes y compañeros, en la búsqueda del conocimiento. Tiene capacidad de compartir experiencias, relacionarse con los demás, trabajar en equipo. Su nivel de aspiración le motiva en cuanto a la cantidad e intensidad de su aprendizaje. Es capaz de desarrollar y utilizar el pensamiento lógico.

Se espera de un estudiante de esta modalidad que pueda tomar sus propias decisiones, organizar y decidir su ritmo de avance, conocer algunas técnicas y procedimientos para estudiar mejor, que se relacione con otros que tengan intereses comunes que sea capaz de desarrollar un proceso de autoevaluación. 
En cuanto a las interacciones que realiza, están relacionadas con los materiales que propone el sistema, con bibliografía, con docentes, con recursos didácticos varios, en contrapartida solicita que el sistema le diga qué le va a ofrecer, porqué y cómo incluyendo todas aquellas cuestiones que le van a ayudar a entender mejor cuál es la propuesta educativa.

También el asesor o docente debe tener un rol que complemente esto y lo apoye, que no lo obstaculice, debe quedar claro que su función es orientar y promover la interacción, darle orientación al estudiante sobre como organizarse con otros compañeros y como trabajar de manera conjunta. EI docente también puede desarrollar y apoyar mejores ambientes de aprendizaje a través de la planeación de los contenidos, generando propuestas tecnológicas, asesorando cuando se requiera su apoyo, proponiendo al estudiante instrumentos de evaluación con propósitos de acreditación, etc. Debe ser, en pocas palabras, un facilitador de los procesos de aprendizaje, que apoye y vaya contribuyendo a esta formación del estudiante que se apropia y se responsabiliza de su propio proceso de aprendizaje.

\section{Conclusión}

Podemos concluir que el uso de la Educación a Distancia e Internet:

- Rompe los límites de la clase tradicional.

- Revaloriza en gran medida el texto escrito y la destreza mental y operativa en los procedimientos de tratamiento de la información.

- Convierte a los usuarios en creadores y consumidores de información.

- Desarrolla actividades colaborativas de enseñanza-aprendizaje entre instituciones y otros actores externos incluyendo los de carácter internacional.

- Requiere realizar adecuaciones o cambios metodológicos, sin dejar de lado la evaluación.

- Promueve criterios y genera habilidades para la discriminación de la información encontrada la cual puede ser muy variada, contradictoria, inadecuada e incluso incomprensible.

- Revaloriza el papel de los docentes como orientadores y mediadores.

- Actualiza las destrezas de los docentes para trabajar en situaciones en que las desigualdades pueden ser muy notorias. 


\section{Bibliografía}

1. SARRAMONA, Jaime. (1993) "El rendimiento en la enseñanza a distancia" Rev. Teoría de la Educación, Vol. V, pp. 127-137

2. CHACÓN, Fabio. (1998) "El nuevo paradigma para el adiestramiento corporativo" Teleconferencia EDUDIST. 20p.

3. CABERO, Julio. (1996) "Nuevas tecnologías, comunicación y educación" en Revista electrónica de tecnología educativa, Palma de Mallorca, España. Num.1 Febrero. 10p.

4. BRANCO VIDA BUSTAMANTE, Silvia. (1999) "Internet y medios tecnológicoas: el ser humano en busca del mensaje" Petrópolis, 6p.

5. SANCHO GIL, Juana ma. (1999) "EEl medio es el mensaje o el mensaje es el medio? El caso de las tecnologías de la información y la comunicación" en Revista Pixel-Bit. No. 4 10p.

6. UNESCO.(1999) "Los docentes, la enseñanza y las nuevas tecnologías" en Informe mundial sobre la educación 1998. Madrid, Santillana/UNESCO pp.78-94

7. BLANCO, Catalina. (1999) "Los desafíos del aprendizaje en el afuera de la enseñanza" en Tercer Simposio Internacional de Educación a Distancia: La educación a distancia y el aprendizaje abierto: Aportes para la construcción de un nuevo paradigma educativo. Mayo 19,20y21 de 1999. Santa Fe de Bogotá, Colombia.pp. 16-23.

8. MORENO ANGARITA, Marisol. "El aprendizaje, un solo motor para la triada más poderosa del siglo 21: información, comunicación y tecnología" en Tercer Simposio Internacional de Educación a Distancia: La educación a distancia y el aprendizaje abierto: Aportes para la construcción de un nuevo paradigma educativo. Mayo 19,20y21 de 1999. Santa Fe de Bogotá, Colombia.pp.95117.

9. COLOM CAÑELLAS, Antonio J. (1998) "Pedagogía tecnológica para la educación a distancia en Los nuevos escenarios educativos y las transformaciones tecnológicas". Patricia Avila comp., ILCE, México, pp. 25-40.

10. BARTOLOMÉ PINA, Antonio R. (1996) "Preparando para un nuevo modo de conocer" en Revista electrónica de tecnología educativa, No. 4 diciembre, 10p.

11. OLLIVIER, BRUNO. (1999) "La universidad y la educación, entre el multimedia y la mundialización ¿Hacia una nueva ideología?" en Tecnología y Comunicación Educativas, ILCEMéxico, No. 29 enero-junio, en prensa.

12. GALLEGO ARRUFAT, MARÍA JESÚS. (1997) "Cuestiones y polémicas en la investigación sobre medios de enseñanza" en La Tecnología Educativa en acción (2ª .ed.), Granada"FORCE. Universidad de Granada, pp.191-208.

13. AVILA MUÑOZ, PATRICIA. (1999). "Aprendizaje con Nuevas Tecnologías" en Revista electrónica de tecnología educativa, 8 p. 